Conclusions The Akha healthcare system is closely related to their religions practice and local wisdom. Any model of health promotion development needs to take into account such issue.

\section{SP1-5 ENDOCRINE, NUTRITIONAL AND METABOLIC DISEASES AS ASSOCIATED CAUSES OF DEATH IN OLDER WOMEN IN RIO DE JANEIRO, BRAZIL}

doi:10.1136/jech.2011.142976m.82

${ }^{1}$ I Mattos, ${ }^{2} \mathrm{P}$ Mattos, ${ }^{1} \mathrm{~L}$ Santiago, * ${ }^{1} \mathrm{~L}$ Luz. ${ }^{1}$ Oswaldo Cruz Foundation, National School of Public Health, Rio de Janeiro, Brazil; ${ }^{2}$ Health Secretariat Rio de Janeiro City, Rio de Janeiro, Brazil

Introduction Changes in lifestyle contributed to an increased incidence and mortality from endocrine, nutritional and metabolic diseases in developing countries. The aim of this study was to evaluate the burden of these diseases as a cause of death in older women in a Brazilian city.

Methods All deaths of women aged 70 years or older, occurring in Rio de Janeiro, between 2003 and 2006, were identified in the Mortality Information System, a population-based nationwide registry. Death certificates with an ICD 10 Chapter IV code (E00-E90) were selected. When the code corresponded to the underlying cause, its subgroup was determined (endocrine, nutritional or metabolic diseases) and the associated causes were ascertained. When these codes corresponded to associated causes, the ICD chapter of the underlying cause was identified.

Results There were 55331 deaths, of which 7686 (13.9\%) had E00E90 codes as underlying (50.4\%) cause. Endocrine diseases predominated among underlying causes. Diabetes was the leading cause of death $(76.4 \%)$ and circulatory and respiratory diseases the main associated causes. As for deaths for other underlying causes, $20.7 \%$ of the circulatory system, $9.5 \%$ of the respiratory system, $6.0 \%$ of the neoplasms and $3.3 \%$ of the infectious diseases had Diabetes as an associated cause.

Conclusion Endocrine diseases, particularly Diabetes, are major causes of mortality in older women in Rio de Janeiro, representing an important public health problem. Urbanisation and socio-cultural influences of the occidental way of life led to changes in dietary patterns and an increased sedentary lifestyle. In order to modify the present patterns, health promotion strategies must be emphasised.

\section{SP1-6 NO EFFECT OF HORMONAL EXPOSURES ON UVEAL MELANOMA}

doi:10.1136/jech.2011.142976m.83

${ }^{1} \mathrm{~T}$ Behrens, ${ }^{* 2,3} \mathrm{~L}$ Kaerlev, ${ }^{4}$ Cree, ${ }^{5} \mathrm{~J} \mathrm{M}$ Lutz, ${ }^{6} \mathrm{~N}$ Afonso, ${ }^{7} \mathrm{M}$ Eriksson, ${ }^{8,9} \mathrm{P}$ Guenel, ${ }^{10} \mathrm{~F}$ Merletti, ${ }^{11,12} \mathrm{M}$ Morales-Suarez-Varela, ${ }^{13} \mathrm{~A}$ Stengrevics, ${ }^{3} \mathrm{~S}$ Sabroe, ${ }^{9,14} \mathrm{D}$ Cyr, ${ }^{11} \mathrm{~A}$ Llopis-Gonzalez, ${ }^{15} \mathrm{G}$ Gorini, ${ }^{7} \mathrm{~L}$ Hardell, ${ }^{16} \mathrm{G}$ Sharkova, ${ }^{1,17} \mathrm{~W}$ Ahrens. ${ }^{1}$ Bremen Institute for Prevention Research and Social Medicine, Bremen, Germany; ${ }^{2}$ Center for National Clinical Databases South, Department of Research and HTA, Odense University Hospital, Odense, Denmark; ${ }^{3}$ Department of Epidemiology, University of Aarhus, Aarhus, Denmark; ${ }^{4}$ Institute of Ophthalmology, University College London, London, UK; ${ }^{5}$ Department of Chronic Disease Epidemiology, National Institute for Cancer Epidemiology and Registration (NICER), ISPM Zürich, University of Zürich, Zürich, Switzerland; ' ${ }^{6}$ Serviço de Oncologia Médica, Instituto Portugues de Oncologia, Porto, Portugal; ' ${ }^{7}$ Department of Oncology, Lund University Hospital, Lund, Sweden; ${ }^{8}$ Inserm, CESP Center for Epidemiology and Population Health, U1018, Environmental Epidemiology of Cancer, Villejuif, France, ${ }^{9}$ University Paris-Sud, UMRS 1018, Villejuif, France: ${ }^{10}$ Unit of Cancer Epidemiology, University of Turin, CERMS and CPO, Piemonte, Italy: ${ }^{11}$ Unit of Public Health and Environmental Care, Department of Preventive Medicine, University of Valencia, Valencia, Spain; ${ }^{12}$ Research group CIBER CB06/02/ 0045 CIBER actions-Epidemiology and Public Health, Valencia, Spain; ${ }^{13}$ Latvia Cancer Registry, Riga, Latvia; ${ }^{14}$ INSERM Unité 687, Villejuif, France; ${ }^{15}$ Epidemiology and Public Health; Environmental and Occupational Epidemiology Unit, ISPO Cancer Prevention and Research Institute, Florence, Italy; ${ }^{16} \mathrm{P}$. Stradin's Clinical University Hospital, Riga, Latvia; ${ }^{17}$ Institute of Medical Informatics, Biometry and Epidemiology, University of Duisburg-Essen, Essen, Germany

Introduction Several studies suggest that hormonal mechanisms may be associated with the development of uveal melanoma.

Objectives To study the association between the risk of uveal melanoma and exposure to hormonal exposures in a case-control study from nine European countries.

Methods Incident cases of uveal melanoma were frequency-matched to population and hospital controls by country, age and sex. Female subjects were asked about their reproductive history, use of menopausal hormone replacement therapy and oral contraceptives Among males, occupational handling of oils while working with transformers or capacitors which contain polychlorinated biphenyls (PCB) was solicited. Unconditional logistic regression analyses were calculated, adjusting for several potential confounders. Analyses were stratified by sex.

Results 293 cases (165 men, 128 women) and 3198 control subjects (2121 men, 1077 women) were interviewed. Among women, no associations were observed with hormonal status variables, intake of hormonal therapy or intake of oral contraceptives. Males showed an increased risk with occupational exposure to transformer/capacitor oils (OR 2.74; Bonferroni-corrected 99.3\% CI 1.07 to 7.02). However, these results were based on few exposed subjects only.

Conclusions The results of this study do not support the hypothesis of a hormonal influence in the carcinogenesis of uveal melanoma Our finding of a potentially increased risk with PCB-containing oils requires further research.

\section{SP1-7 PESTICIDE EXPOSURE IN FARMING AND FORESTRY AND THE RISK OF UVEAL MELANOMA}

doi:10.1136/jech.2011.142976m.84

${ }^{1} \mathrm{~T}$ Behrens, ${ }^{*} \mathrm{E}$ Lynge, ${ }^{3} \mathrm{I}$ Cree, ${ }^{4} \mathrm{~J} \mathrm{M}$ Lutz, ${ }^{5} \mathrm{M}$ Eriksson, ${ }^{6,7} \mathrm{P}$ Guenel, ${ }^{8} \mathrm{~F}$ Merletti, ${ }^{9,10} \mathrm{M}$ Morales-Suarez-Varela, ${ }^{11} \mathrm{~N}$ Afonso, ${ }^{12} \mathrm{~A}$ Stengrevics, ${ }^{13} \mathrm{~J}$ Fevotte, ${ }^{14} \mathrm{~S}$ Sabroe, ${ }^{9,10} \mathrm{~A}$ Llopis-Gonzalez, ${ }^{15} \mathrm{G}$ Gorini, ${ }^{16} \mathrm{~L}$ Hardell, ${ }^{17,18} \mathrm{~S}$ Andreas, ${ }^{1,18} \mathrm{~W}$ Ahrens. ${ }^{1}$ Bremen Institute for Prevention Research and Social Medicine, Bremen, Germany, ${ }^{2}$ Institute of Public Health, University of Copenhagen, Copenhagen, Denmark; ${ }^{3}$ Institute of Ophthalmology, University College London, London, UK; ${ }^{4}$ Department of Chronic Disease Epidemiology, National Institute for Cancer Epidemiology and Registration (NICER), ISPM Zürich, Zürich, Switzerland; ${ }^{5}$ Department of Oncology, University Hospital, Lund, Sweden; ${ }^{6}$ Inserm, CESP Center for Epidemiology and Population Health, U1018, Environmental Epidemiology of Cancer, Villejuif, France; ${ }^{7}$ University Paris-Sud, UMRS 1018, Villejuif, France; ${ }^{8}$ Unit of Cancer Epidemiology, University of Turin, CERMS and CPO, Piemonte, Italy; ${ }^{9}$ Unit of Public Health and Environmental Care, Department of Preventive Medicine, University of Valencia, Valencia, Spain; ${ }^{10}$ Research Foundation. University Hospital Dr. Peset, Valencia, Spain; ${ }^{11}$ Serviço de Oncologia Médica, Instituto Portugues de Oncologia, Porto, Portugal; ${ }^{12}$ Latvia Cancer Registry, Riga, Latvia; ${ }^{13}$ Institut de Veille Sanitaire (InVS), Département, Santé-Travail (DST), S. Maurice, France; ${ }^{14}$ Department of Epidemiology, University of Aarhus, Aarhus, Denmark; ${ }^{15}$ Epidemiology and Public Health; Environmental and Occupational Epidemiology Unit-ISPO Cancer Prevention and Research Institute, Florence, Italy; ${ }^{16}$ Department of Oncology, Lund University Hospital, Lund, Sweden; ${ }^{17}$ Institute of Clinical Epidemiology, University of Halle-Wittenberg, Halle, Germany; ${ }^{18}$ Institute of Medical Informatics, Biometry and Epidemiology, University of Duisburg-Essen, Essen, Germany

Introduction Since pesticides are disputed risk factors for uveal melanoma, we studied the association between occupational pesticide exposure and uveal melanoma risk in a case-control study from nine European countries.

Methods Incident cases of uveal melanoma and population as well as hospital controls were included and frequency-matched by country, 5-year age groups and sex. Self-reported exposure was quantified with respect to duration of exposure and pesticide 